\title{
Impact of Relaxation and Group Psycho-Education on Health Outcomes Using Integrated Care Models and Quality Improvement
}

\author{
Martha Okafor ${ }^{1}$, Victor Ede ${ }^{1}$, Rosemary Kinuthia ${ }^{1}$, Debbie Strotz ${ }^{2}$, Cathryn Marchman ${ }^{3}$, \\ Scott Weeks ${ }^{4}$, David Satcher ${ }^{1}$ \\ ${ }^{1}$ Satcher Health Leadership Institute, Division of Behavioral Health, Morehouse School of Medicine, Atlanta, \\ GA, USA \\ ${ }^{2}$ Rehabilitation and Recovery Services, Cobb and Douglas County Community Services Board, Austell, GA, USA \\ ${ }^{3}$ Advocacy and Compliance, Mercy Care, Atlanta, GA, USA \\ ${ }^{4}$ Cobb and Douglas County Community Services Board, Austell, GA, USA \\ Email: vede@msm.edu
}

Received 6 October 2014; revised 3 November 2014; accepted 1 December 2014

Copyright (C) 2014 by authors and Scientific Research Publishing Inc.

This work is licensed under the Creative Commons Attribution International License (CC BY). http://creativecommons.org/licenses/by/4.0/

(c) () Open Access

\section{Abstract}

The purpose of this Integrated Care Practice Change and Quality Improvement (ICPCQI) initiative was to evaluate the impact of wellness and relaxation and group psycho-education on health outcomes in an integrated care setting. Individuals diagnosed with mental illness and a co-occurring chronic medical condition participated in the ICPCQI initiatives which were run by peer support and wellness experts in an integrated care setting over the one-year project implementation period. Evaluation of outcome measures revealed an overall decline of $8.3 \%(p<0.0001)$ and $7.3 \%$ $(p<0.0001)$ in the average systolic and diastolic blood pressure respectively. There was a $12.3 \%$ $(p=0.02)$ reduction on the average PHQ-9 scores. GAD-7 ( $p=0.9)$ scores had a mean reduction of $1.5 \%$. These preliminary results suggest that the evidence-based ICPCQI initiatives positively impact health outcomes among individuals with mental illness and chronic medical conditions.

\section{Keywords}

Integrated Care, Relaxation, Group Psycho-Education, Health Outcomes, Quality Improvement, Peer Specialist, Wellness, Primary Healthcare, Behavioral Health

\footnotetext{
${ }^{*}$ Corresponding author.
}

How to cite this paper: Okafor, M., Ede, V., Kinuthia, R., Strotz, D., Marchman, C., Weeks, S. and Satcher, D. (2014) Impact of Relaxation and Group Psycho-Education on Health Outcomes Using Integrated Care Models and Quality Improvement. Open Journal of Preventive Medicine, 4, 887-896. http://dx.doi.org/10.4236/ojpm.2014.412100 


\section{Background}

Approximately one fourth of adults in the United States have a mental illness, and nearly half will develop at least one mental illness during their lifetime [1] [2]. Mental illness is an important public health problem, both in its own right and because the condition is associated with other chronic diseases and their resulting morbidity and mortality [3]. Mental illness exacerbates morbidity from the multiple chronic diseases with which it is associated, including cardiovascular disease, diabetes, obesity, asthma, epilepsy and cancer [4]-[6]. This increased morbidity is a result of lower use of medical care and treatment adherence for concurrent chronic diseases and higher risk for adverse health outcomes [7]-[9]. Furthermore, mental illness cost the United States an estimated $\$ 300$ billion annually, which includes approximately $\$ 193$ billion from lost earnings and wages and $\$ 24$ billion in disability benefits [10] and $\$ 100$ billion in health-care expenditures [11]. There is therefore, a clear need for, integrative, cost-effective interventions that address mental and physical health issues.

The Satcher Health Leadership Institute's (SHLI) goal in this pilot was to improve health outcomes among individuals with behavioral health needs and co-morbid medical conditions, while reducing cost, through the implementation of a specific Integrated Care Practice Change and Quality Improvement (ICPCQI) initiative within an evidence-based framework. SHLI in collaboration with selected Community Health Centers implemented innovative integrated primary health and behavioral health care models which incorporated peer specialists, behavioral health clinicians, relaxation techniques and group psycho-education in community health settings. The expansion of peer specialists, into the mental health system, recognizes that the experience of recovery from mental health problems is a valuable source of knowledge and that peer relationships are a powerful and unique source of support for people with mental health conditions [12] [13]. Peer specialists have a lived mental health experience and an empathetic understanding of what a client is going through, allowing them to work with clients by utilizing this shared experience [14]. As a care team member, the peer specialist can bring first-hand knowledge of the integrated care system [15] and how to access services working with both clients and treatment teams.

Group psycho-education therapy provided by a behavioral health specialist or behavioral health clinician was utilized in this ICPCQI pilot. Psycho-education is the provision of clinically-relevant information to address psychological variables in individuals with a physical or psychological disorder [16]. Group psycho-education can be carried out through face-to-face interaction or through audio-visual aids [16]. A meta-analysis by Donker et al. (2006) revealed that brief passive psycho-educational interventions for depression and psychological distress can reduce symptoms.

Relaxation interventions (such as meditation, yoga, muscle relaxation, abdominal breathing and guided imagery) have been widely used among patients in various health settings [16]. In the state of relaxation, individuals retreat mentally from their surroundings to decrease tension and induce calm/relaxed feelings [17]. The relaxation response undoes the stress response, leading to reduced sympathetic nervous arousal and the activation of the parasympathetic nervous system. This activation results in decreased muscle tension, blood pressure, heart rate and respiration rate [18]. The relaxation response also activates the limbic system, which might help alleviate depression and anxiety.

This pilot adopted 2 integrated care practice models: the co-location model and the disease management model. The co-location model uses specialty mental health clinicians who provide services at the same site as primary care [19]. The co-location model also incorporated a psycho-education component provided by a behavioral health specialist. The ICPCQI pilot also utilized the disease management (or chronic care) model, which is an integrated system of interventions to optimize functioning of patients and impact the overall cost of disease burden [20]. The model incorporated a mindfulness expert and certified peer specialist into the chronic care model. This disease management model was adopted because psychological stress accompanies chronic illnesses - a problem this project aimed to address.

\section{Methods}

\subsection{Participants and Procedures}

This quality improvement and practice change initiative was developed through a partnership between Kaiser Permanente and the SHLI and approved by the Institutional Review Board at Morehouse School of Medicine, Atlanta. The ICPCQI evaluation used a logic model approach in monitoring, tracking, and measuring the impact 
of two community health centers projects in Georgia namely: Cobb Community Service Board (The Circle) and Saint Joseph's Mercy Care Services (The Gateway Clinic). We examined both the process of implementing ICPCQI at the two community health centers, as well as the outcomes. Process evaluation was done using: the SHLI Collaborative Integrated Care Progress Report - a quarterly report that included milestone status, impact, and Plan Do Study Act \& Account [PDSAA] completion update; and Community of Practice process, which includes SHLI monthly collaborative teleconferences and on-site learning academies.

The Community of Practice (CoP) for the ICPCQI was formed by five community health centers who engaged in collective shared learning with a common interest and commitment to improve health outcomes of their patients with both medical and behavioral health problems using an integrated care practice model. These five community health centers shared, learned and implemented changes in their health centers to transform their practice to be more integrated for three years. In their first year, they functioned as a learning community whereby they learned more about integrated care models and discovered ways to improve their practices to become more integrated by implementing small steps of changes that were studied. Lessons learned from their collective small steps of changes were shared on a weekly conference call with all sites.

In the second year, the five community health centers formed a CoP that connects participants from each site to interact, share information, personal stories and experiences of possible solutions to implementing integrated care practice that ranged from transforming the reception and front desk area, to scheduling, to triage, and warm hands-off practices. They defined a specific domain for their continuous learning in the second year, i.e. to focus on the use of quality improvement practices to advance integrated care model in their centers. The SHLI designed a CoP learning experience that required proposed quality improvement projects with specific interventions, measures and expected results. Also, SHLI facilitated a collective learning experience for the CoP on a monthly basis to enable dialogue and shared learning of developed and tested interventions and tools; emerging discoveries of solutions to challenges of integrating behavior health and primary care; and rotational on-site visits of the sites to observe and learn about their QI projects. To stimulate free flow of ideas, capture promising practices, and strengthen collaborative learning, the $\mathrm{CoP}$ members were engaged in every other month learning academies where evidence-based knowledge, promising practices and improvements were discussed to develop both leadership and clinical skills. The benefits of the CoP resulted in leadership development, collaborative and free sharing of ideas and exchange of information, which accelerated spread of promising practices that are adapted and replicated in different sites to transform practices in order to improve patient health outcomes.

The CoP sites also used the four stages of the Plan-Do-Study-Act (PDSA) cycle. The PDSA is sometimes called Deming wheel and defined as "a systemic series of small steps for gaining valuable learning and knowledge for the continual improvement of a product or process" [21]. Using this model of improvement, the CoP sites: Plan - the specific practice change (ICPCQI) to be implemented with goals, action steps and results metrics; Do - carry out the ICPCQI specific action step; Study - observe the action, collect data before and after the ICPCQI and reflect on what works and areas for improvement as lessons learned; Act-adjust subsequent actions steps, re-align goals and plan the next change or full implementation.

\subsection{Integrated Behavioral and Primary Healthcare Sites}

The Circle provides outpatient services to individuals with mental health and substance abuse challenges. This site implemented a relaxation and wellness program to address hypertension and anxiety. 37 patients with anxiety/stress disorder and co-occurring hypertension participated in the program. There were 2 cohorts (17 and 20 participants each). Participants were expected to attend weekly sessions for three months. Of the 37 participants, only 20 completed pre and post GAD-7 evaluations. Using a best practice curriculum that consists of guided imagery and meditation techniques, a mindfulness and relaxation expert trained patients with knowledge, skills, and techniques to develop new coping mechanisms outside of the group. The relaxation expert also coordinated the group sessions. Meditation techniques were taught to the patients to help them focus and keep their minds from becoming cluttered with negative thoughts while promoting health. A certified peer specialist (CPS) monitored and was available to assist with mindfulness and guided relaxation during the non-group hours. The peer support also helped to retain the participants in the groups. These CPSs receive training through the Georgia Certified Peer Specialist Project to assist their peers in skills building, goal setting, problem solving, conducting Recovery Dialogues, setting up and sustaining mutual self-help groups, and in helping their peers build their own self-directed recovery tools [22]. Pre and post blood pressure readings were taken. A self-administered an- 
xiety measure (GAD-7) was administered at the start and end of the group session.

Saint Joseph's Mercy Care Services (SJMCS) provides primary healthcare services to the homeless and underserved in metro Atlanta through a combination of fixed and mobile clinic sites. A board certified psychiatrist was co-located in the gateway clinic. The psychiatrist provided mental health care to patients, coordinated referrals, and trained providers using a standardized integrated physical and behavioral health care didactics curriculum. SJMCS/Gateway implemented five 8-week psycho-educational Hypertension/Depression groups that served a total of 50 homeless patients. The participants had comorbid diagnoses of depression and hypertension and were treated at SJMCS. Participants received a free round trip Marta public transportation card for attending each session, as well as a healthy snack to enjoy during the group sessions. Improvements in health outcomes among patients with mental illness and co-occurring chronic medical conditions were measured using clinical indicators. Changes in Hypertension were measured with blood pressure recordings; depression was assessed using the PHQ-9 scale, and change in knowledge was measure by pre and post-tests. These metrics were captured before and after participants started the program.

Outcome evaluation examined data collected using quantitative assessment tools tailored to the two initiatives. We used paired $t$ test statistic to determine changes in mean pre and post blood pressures, PHQ-9 and GAD-7 scores. Data analysis was facilitated by SPSS Inc. (PASW Statistics 18). Both quantitative and qualitative data were provided from September 2012 to October 2013.

\section{Results}

Table 1 presents the pre-intervention (before integration) and post-intervention (after integration) quality metrics. The blood pressure quality metric was categorized into systolic and diastolic. Overall, there was a decline in the quality metrics following the implementation of the ICPCQI. When we compared the average pre-intervention systolic blood pressure with post-intervention, there was a statistically significant decrease of 11.26 in the mean systolic blood pressure (Figure 1), which may be attributed to implementation of integrated behavioral care. The average diastolic blood pressure before the program was 85.1 and 78.8 after implementation of the ICPCQI (Figure 2). This project showed a 7.3\% decline in the average diastolic blood pressure. The average PHQ-9 score decreased significantly by $12.3 \%$ after initiation of the program with a mean depression score of 12.4 before and 10.9 after the start of the program, $p=0.02$ (Figure 3).

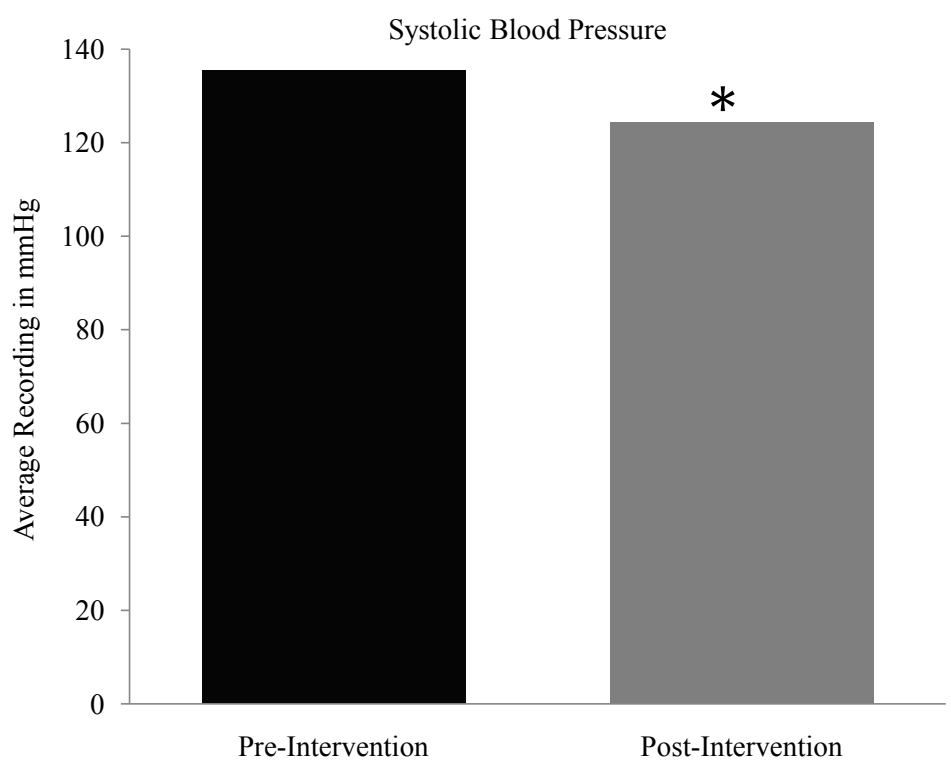

Figure 1. Comparison of average systolic blood pressure recordings among patients who participated in the Integrated Care Practice Change and Quality Improvement project at The Circle and Gateway clinics $(\mathrm{n}=87)$ : Georgia, September 2012 to October 2013. *Represents a $p$ value $<$ 0.0001 . 


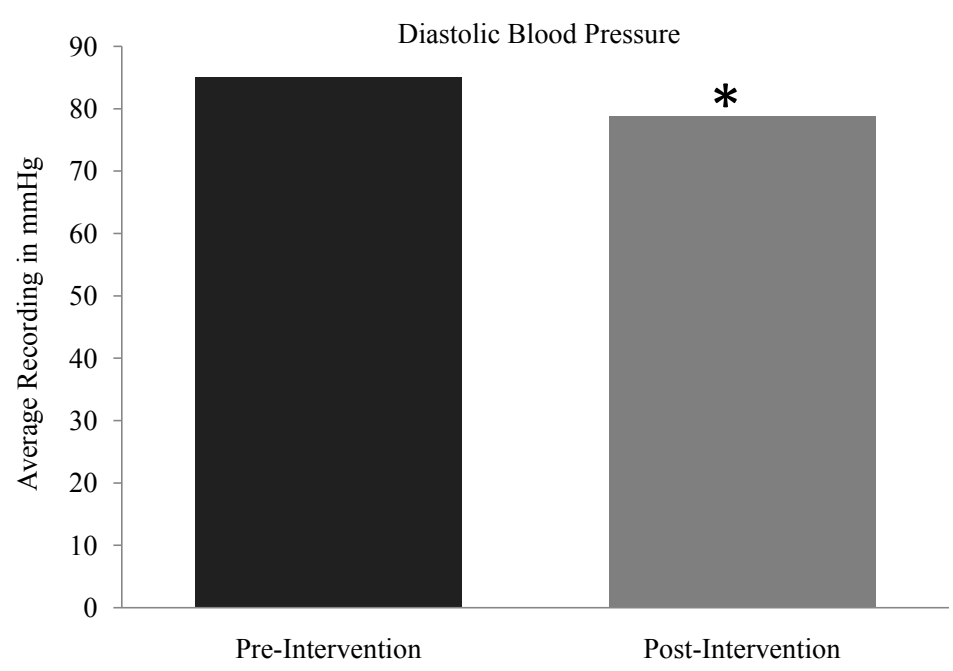

Figure 2. Comparison of average diastolic blood pressure recordings of patients who participated in the integrated care practice change and quality improvement project at the circle and gateway clinics $(n=87)$ : Georgia, September 2012 to October 2013. ${ }^{*}$ Represents a $p$ value $<0.0001$.

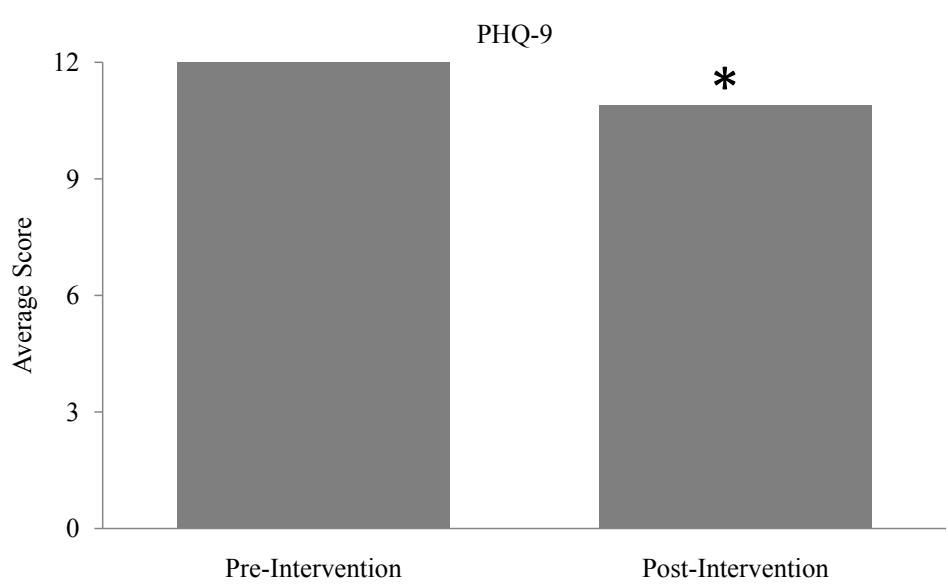

Figure 3. Comparison of average PHQ-9 scores among patients who participated in the integrated care practice change and quality improvement project at gateway clinic $(n=50)$ : Georgia, September 2012 to October 2013. ${ }^{*}$ Represents a $p$ value $<0.05$.

Table 1. Integrated care practice change and quality improvement metrics, data from the circle and gateway clinics $(\mathrm{n}=$ 87): Georgia, September 2012 to October 2013.

\begin{tabular}{cccccc}
\hline Metrics & Pre-Intervention & Post-Intervention & \multicolumn{2}{c}{ Mean Difference } & $p$ Value \\
\hline PHQ-9 & 12.4 & 10.9 & 1.53 & $(12.3 \%$ decrease $)$ & 0.02 \\
Blood Pressure & & & & & \\
Systolic & 135.5 & 124.3 & 11.26 & $(8.3 \%$ decrease $)$ & $<0.0001$ \\
Diastolic & 85.1 & 78.8 & 6.28 & $(7.3 \%$ decrease $)$ & $<0.0001$ \\
GAD-7 & 8.87 & 8.73 & 0.13 & $(1.5 \%$ decrease $)$ & 0.9
\end{tabular}

Note: PHQ-9 = Patient Health Questionnaire; GAD-7 = Generalized Anxiety Disorder; GAD-7 data were collected for 20 adult participants at the circle. 


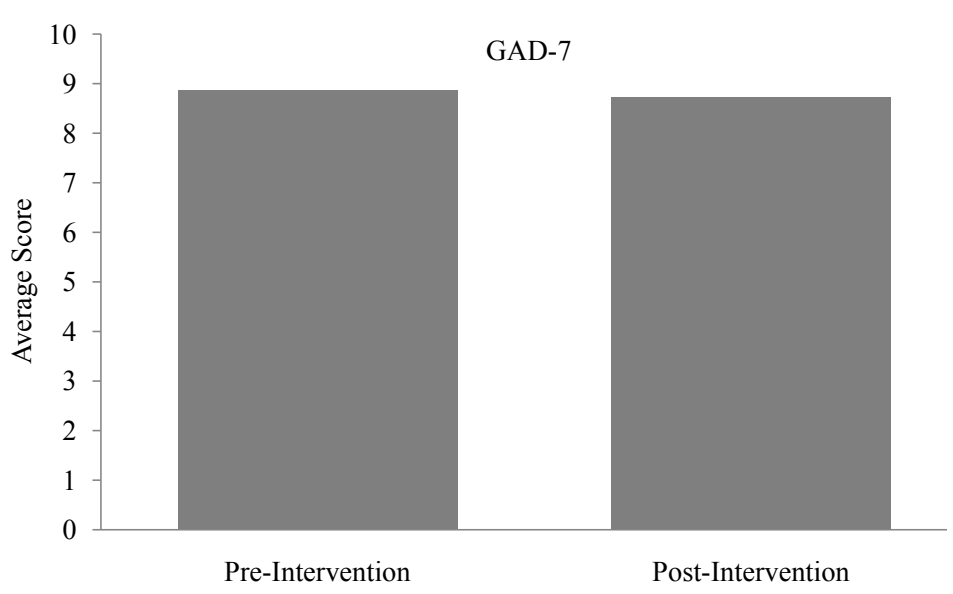

Figure 4. Comparison of average GAD-7 scores among patients who participated in the integrated care practice change and quality improvement project at the circle $(\mathrm{n}=20)$. $*$ Represents a $p$ value $<0.05$.

When comparing the average GAD-7 score before and after implementation of the ICPCQI, there was a 1.5\% reduction in the mean GAD-7. However, this decrease was not statistically significant. The average GAD-7 score before the start of the program was 8.87 and 8.73 after the program was initiated (Figure 4). Among SJMCS participants, $65 \%$ attended all group sessions. Twenty-three (45.8\%) participants completed both pre and post PHQ-9 evaluations, 35\% completed pre and post knowledge tests and $85 \%$ had pre and post blood pressure documented. $83 \%$ of Cobb CSB participants had pre and post blood pressure readings documented.

\section{Discussion}

These findings suggest that the SHLI Integrated Care Practice Change and Quality Improvement Initiatives were generally successful in achieving its goals. In particular:

\subsection{Improving Health Outcomes}

Improving health outcomes and potentially reducing health care costs were successfully accomplished. Through the adoption of integrated practice changes and quality improvement strategies, community health centers successfully improved both physical and mental health. Although a robust evaluation that includes cost analysis and return on investment was not done, there is evidence to support potential reduction of healthcare costs using similar integrated care models.

\subsection{Improved Culturally Appropriate Integrated Care}

Implementation of culturally appropriate strategies, process and practice are needed for providing integrated care to people with co-morbid conditions, who are of minority population, or have complex behavioral health needs. SHLI in collaboration with the clinic sites successfully implemented evidence-based culturally appropriate integrated care models in a Federally Qualified Health Center and a community mental health center. The majority of people served by these clinics were minorities, who had a mental illness and a co-morbid chronic medical condition.

\subsection{Enhanced Capacity Building}

Support capacity building of continuous quality improvement project to increase access to integrated care services, and advance innovation, leadership, and policy was needed to promote behavioral health equity. The SHLI community of practice (COP) collaborative academy helped to strengthen the capacity of the integrated care sites to develop more competencies in determining priorities and implementing quality improvements, such as PDSA, sharing, learning and accelerating emerging best practices, and building a sustainable team and quality improvement infrastructure to increase access to mental health and primary health among under-served popu- 
lations. This has led to the adoption and expansion of innovative integrated care practice change and quality improvement models among the health centers involved in the COP besides the two sites being discussed in this article.

\subsection{Affordable Care Act Opportunities}

There are opportunities for Peer Support in the Affordable Care Act (ACA). Peer support services promote several key objectives of the ACA. Integrated into community-based care, peer support services have the potential to improve the quality of healthcare delivery, lower healthcare expenditures, and reduce health disparities. The community health worker (CHW) model has been shown to be effective in delivering peer support services in a variety of settings. The ACA formally recognizes the role of $\mathrm{CHWs}$ and offers several funding opportunities for CHW programs.

\subsection{Improved Quality of Life}

Peer Specialists may provide the critical impetus to help other peers improve their quality of life, recovery and life-spans. Peers working in integrated settings helped persons served to develop skills, abilities, and potential to achieve personal goals. The peer wellness approach adopted by Cobb CCSB empowered other peers to assume personal responsibility and be proactive in the preservation of his or her own health. Through guided imagery, participants learned the connection between the mind and body. The participants were able to go to a safe, calming place in their minds and their physical bodies reacted as if it were in that safe place.

\subsection{Cost Savings}

The use of peer support and relaxation and mindfulness experts in community heath settings is cost-effective. Some of the appeal of hiring peer specialists is that they are less expensive than licensed professionals, such as nurses, nutritionists, and diabetes educators. The peer specialist community involved in this initiative has certified members and they provided "more services for less cost". A glass ceiling often restricts peers' career advancement. Furthermore, the overall cost of using a relaxation and mindfulness expert in an integrated care setting may likely be more cost effective in comparison to services provided by a psychiatrist or physician in that setting.

\subsection{Collaboration Building}

The use of CoP was an important and crucial element of the collaborative process that supported the results evident in the participating sites. The $\mathrm{CoP}$ connected the sites to build more knowledge, understanding and insights about ways to improve their practices to be more integrated. It allowed the members to form new partnerships, problem-solve, request and freely share information, explore new possibilities and create opportunities to discover shared solutions to their challenges. The monthly engagement fostered sharing of promising tools, and replicating or adapting promising interventions; learning of evidence-based practices and closing identified gaps.

\section{Limitations}

Several lessons emerged from the process of implementing the ICPCQI and evaluation that maybe useful for other similar initiatives:

\subsection{Retention of Patients in Integrated Behavioral Health Program/Attrition Rates}

Retaining all participants in the group psycho-educational programs and relaxation and wellness cohorts were challenging. Factors that contributed to this ranged from patients transitioning from various housing situations, to lack of transportation among homeless populations, to having closed groups. This was addressed by incorporating more incentives to aid transportation, increasing marketing, and changing from closed groups to open groups.

\subsection{Confounding Factors}

Although some promising work has been accomplished, the results from the analysis of the ICPCQI alone do 
not conclude for certain that the specific interventions resulted in the improvement of health outcomes. Therefore, further studies that explore the impact of potential confounders such as pharmacotherapy and medication adherence are essential.

\subsection{Policy Implications of Integrated Care}

A major policy implication of integrated care is its alignment with the Patient Protection and Affordable Care Act (ACA) and its requirement for Patient-Centered Medical Home (PCMH) [23]. For healthcare centers to fully implement and operate as a PCMH, it has to provide a comprehensive health care to respond to their patients' presenting health needs, which include both primary and behavioral health services.

In addition, the ACA requires healthcare settings to participate in comparative effectiveness of how they manage their patients' health status. This encourages healthcare providers to go beyond treating the incidence of diseases to addressing the wellbeing, preventative and burden of population health with the goals of implementing and accelerating evidence-based practices, improving quality clinical care and patients' outcomes, while reducing health disparities and healthcare cost. There is emerging evidence showing that integrated care can be utilized to meet this requirement, such as IMPACT model [24] [25]. In addition, emerging results from our demonstration sites support current evidence indicating that integrated care can be applied as a comparative effective approach for improving health outcomes and overall population health.

Also, integrated care practice based on its inherent requirement for multi-disciplinary health professionals to co-manage the complexities of the patients' co-morbid medical and behavioral health needs, supports the implementation of the Centers for Medicare \& Medicaid Services' (CMS) meaningful-use provisions and incentive payments. To be eligible for the incentive payments, providers will have to demonstrate their use of an electronic system that includes electronic health records and drug-drug interaction, allergy, and the capability to generate and transmit patients health information, treatment plans and prescriptions electronically. The policy questions are always concentrated on the cost of investing in such health information systems that must be integrated to foster both the effectiveness and efficiency of healthcare and patient outcomes. Although, emerging evidence shows that integrated care plan offer benefits to the patients, healthcare providers, organizations, and significantly supports the intent of the ACA [26] [27], the federal and state governmental entities are yet to ensure that we have sufficient policy requirements to cover and compensate for this care model.

The ICPCQI described above linked the organizational and process improvement, partnership structure and outcomes measures of the quality of the participants' relational engagement in $\mathrm{CoP}$, which contributed to their sharing of emerging best practices to increase access and utilization of health care and services. The demonstration of the ICPCQI also showed that the integrated care services provided on the same day to patients were not reimbursable by the Georgia State healthcare insurance programs.

The limitation of the reimbursement of "same day" services negates the intent of providing quality health care to reduce cost and improve patients' outcomes. To successfully implement the ACA, policy needs to be aligned with this evidence-based practice that contributes to the accomplishment of desired health outcomes. Such change in policy is crucial for supporting the use of multidisciplinary team of health professionals in understanding and improving health and wellness of individual patients and the population as a whole. The absence of such responsive policy to support integrated care practice, process, structure and treatment will ultimately negate the real intent and expectations of the ACA and PCMH as people with complex co-morbid primary and behavioral health care needs continue to use emergency care, inappropriate in-patient care and inadequate care at their PCMH. As a result, there is an urgent need to better understand the costs [financial, human suffering and life] of integrated care practice and approach and use the evidence to inform policy changes that will result in coverage of essential "billable codes" as part of the basic mandated health benefits in every state health plan.

\section{Conclusions}

The findings of this quality improvement initiative highlight the important role that integration of care may have in improving behavioral health outcomes in individuals with chronic medical illness.

There is a higher demand for behavioral health services and with the rising costs of healthcare and shortage of behavioral health providers, implementing cost saving services such as the use of peer support specialists and wellness experts will not only address the shortage of behavioral health providers but could also improve health outcomes of individuals with mental illness and chronic medical conditions. Further research incorporating the 
impact of the discussed confounders is necessary to determine whether there is a correlation between group psycho-educational and relaxation/wellness services and improved healthcare outcomes.

\section{Acknowledgements}

Integrated Care Practice Change and Quality Improvement (ICPCQI) initiative was funded by grants from Kaiser Permanente and Georgia Department of Behavioral Health and Developmental Disabilities (DBHDD). We also thank the patients, staff, and providers at Cobb Community Service Board (The Circle) and Saint Joseph's Mercy Care Services (The Gateway Clinic) for their participation in the ICPCQI.

\section{Conflict of Interest Statement}

The authors do not have any conflicts of interest to report.

\section{References}

[1] Kessler, R.C., Berglund, P., Demler, O., Jin, R., Merikangas, K.R. and Walters, E.E. (2005) Lifetime Prevalence and Age-of-Onset Distributions of DSM-IV Disorders in the National Comorbidity Survey Replication. Archives of General Psychiatry, 62, 593-602. http://dx.doi.org/10.1001/archpsyc.62.6.593

[2] Kessler, R.C., Berglund, P., Demler, O., et al. (2003) The Epidemiology of Major Depressive Disorder: Results from the National Comorbidity Survey Replication (NCS-R). Journal of American Medical Association, 289, 3095-3105. http://dx.doi.org/10.1001/jama.289.23.3095

[3] Reeves, W.C. and Strine, T.W., Pratt, L.A., et al. (2011) Mental Illness Surveillance among Adults in the United States. MMWR Surveillance Summaries, 60, 1-29.

[4] Kessler, R.C., Heeringa, S., Lakoma, M.D., Petukhova, M., Rupp, A.E., Schoenbaum, M., et al. (2008) Individual and Societal Effects of Mental Disorders on Earnings in the United States: Results from the National Comorbidity Survey Replication. American Journal of Psychiatry, 165, 703-711.

[5] Chapman, D.P., Perry, G.S. and Strine, T.W. (2005) The Vital Link between Chronic Disease and Depressive Disorders. Preventing Chronic Disease, 2 , A14.

[6] Lichtman, J.H., Bigger Jr., J.T., Blumenthal, J.A., et al. (2008) Depression and Coronary Heart Disease: Recommendations for Screening, Referral, and Treatment: A Science Advisory from the American Heart Association Prevention Committee of the Council on Cardiovascular Nursing, Council on Clinical Cardiology, Council on Epidemiology and Prevention, and Interdisciplinary Council on Quality of Care and Outcomes Research: Endorsed by the American Psychiatric Association. Circulation, 118, 1768-1775. http://dx.doi.org/10.1161/CIRCULATIONAHA.108.190769

[7] Keyser, D.J., Houtsinger, J.K., Watkins, K. and Pincus, H.A. (2008) Applying the Institute of Medicine Quality Chasm Framework to Improving Health Care for Mental and Substance Use Conditions. Psychiatrics Clinic of North America, 31, 43-56. http://dx.doi.org/10.1016/j.psc.2007.11.002

[8] Broadbent, E., Kydd, R., Sanders, D. and Vanderpyl, J. (2008) Unmet Needs and Treatment Seeking in High Users of Mental Health Services: Role of Illness Perceptions. Australian \& New Zealand Journal of Psychiatry, 42, 147-153. http://dx.doi.org/10.1080/00048670701787503

[9] Levinson, D., Karger, C.J. and Haklai, Z. (2008) Chronic Physical Conditions and Use of Health Services among Persons with Mental Disorders: Results from the Israel National Health Survey. General Hospital Psychiatry, 30, $226-232$. http://dx.doi.org/10.1016/j.genhosppsych.2008.02.007

[10] Insel, T.R. (2008) Assessing the Economic Costs of Serious Mental Illness. American Journal of Psychiatry, 165, 663665. http://dx.doi.org/10.1176/appi.ajp.2008.08030366

[11] Mark, T.L., Levit, K.R., Buck, J.A., Coffey, R.M. and Vandivort-Warren, R. (2007) Mental Health Treatment Expenditure Trends, 1986-2003. Psychiatric Services, 58, 1041-1048. http://dx.doi.org/10.1176/appi.ps.58.8.1041

[12] Gates, L.B. and Akabas, S.H. (2007) Developing Strategies to Integrate Peer Providers into the Staff of Mental Health Agencies. Administration and Policy in Mental Health and Mental Health Services Research, 34, 293-306. http://dx.doi.org/10.1007/s10488-006-0109-4

[13] Chinman, M., Young, A.S., Hassell, J. and Davidson, L. (2006) Toward the Implementation of Mental Health Consumer Provider Services. Journal of Behavioral Health Services \& Research, 33, 176-195. http://dx.doi.org/10.1007/s11414-006-9009-3

[14] Mowbray, C.T., Moxley, D.P., Thrasher, S., Bybee, D. and Harris, S. (1996) Consumers as Community Support Providers: Issues Created by Role Innovation. Community Mental Health Journal, 32, 47-67. http://dx.doi.org/10.1007/BF02249367 
[15] Paulson, R., Herinckx, H., Demmler, J., Clarke, G., Cutler, D. and Birecree, E. (1999) Comparing Practice Patterns of Consumer and Non-Consumer Mental Health Service Providers. Community Mental Health Journal, 35, 251-269. http://dx.doi.org/10.1023/A:1018745403590

[16] Donker, T., Griffiths, K.M., Cuijpers, P. and Christensen, H. (2009) Psychoeducation for Depression, Anxiety and Psychological Distress: A Meta-Analysis. BMC Medicine, 7, 79. http://dx.doi.org/10.1186/1741-7015-7-79

[17] DeMarco-Sinatra, J. (2000) Relaxation Training as a Holistic Nursing Intervention. Holistic Nursing Practice, 14, 30-39. http://dx.doi.org/10.1097/00004650-200004000-00007

[18] Shah, L.B., Klainin-Yobas, P., Torres, S. and Kannusamy, P. (2014). Efficacy of Psychoeducation and Relaxation Interventions on Stress-Related Variables in People with Mental Disorders: A Literature Review. Archives of Psychiatric Nursing, 28, 94-101.

[19] Robinson, P.J. and Strosahl, K.D. (2009) Behavioral Health Consultation and Primary Care: Lessons Learned. Journal of Clinical Psychology in Medical Settings, 16, 58-71. http://dx.doi.org/10.1007/s10880-009-9145-z

[20] Gabbay, R.A., Bailit, M.H., Mauger, D.T., Wagner, E.H. and Siminerio, L. (2011) Multipayer Patient-Centered Medical Home Implementation Guided by the Chronic Care Model. Joint Commission Journal on Quality and Patient Safety, 37, 265-273.

[21] The PDSA Cycle. The W.Edwards Deming Institute Web Site. https://www.deming.org/theman/theories/pdsacycle

[22] Georgia Certified Peer Specialist Project. Georgia Certified Peer Specialist Project Web Site. http://www.gacps.org/Home.html

[23] Viron, M., Zioto, K., Schweitzer, J. and Levine, G. (2014) Behavioral Health Homes: An Opportunity to Address Healthcare Inequities in People with Serious Mental Illness. Asian Journal of Psychiatry, 10, 10-16. http://dx.doi.org/10.1016/j.ajp.2014.03.009

[24] Unutzer, J., Katon, W., Williams Jr., J.W., Callahan, C.M., Harpole, L., Hunkeler, E.M., et al. (2001) Improving Primary Care for Depression in Late Life: The Design of a Multicenter Randomized Trial. Medical Care, 39, 785-799. http://dx.doi.org/10.1097/00005650-200108000-00005

[25] Van Leeuwen, W.E., Unutzer, J., Lee, S. and Noel, P.H. (2009) Collaborative Depression Care for the Old-Old: Findings from the IMPACT Trial. American Journal of Geriatric Psychiatry, 17, 1040-1049. http://dx.doi.org/10.1097/JGP.0b013e3181b4bf08

[26] Laderman, M. and Mate, K. (2014) Integrating Behavioral Health into Primary Care. Healthcare Executive, $29,74-77$. http://www.ihi.org/resources/Pages/Publications/IntegratingBehavioralHealthPrimaryCare.aspx

[27] Kuramoto, F. (2014) The Affordable Care Act and Integrated Care. Journal of Social Work in Disability \& Rehabilitation, 13, 44-86. http://dx.doi.org/10.1080/1536710X.2013.870515 
Scientific Research Publishing (SCIRP) is one of the largest Open Access journal publishers. It is currently publishing more than 200 open access, online, peer-reviewed journals covering a wide range of academic disciplines. SCIRP serves the worldwide academic communities and contributes to the progress and application of science with its publication.

Other selected journals from SCIRP are listed as below. Submit your manuscript to us via either submit@scirp.org or Online Submission Portal.
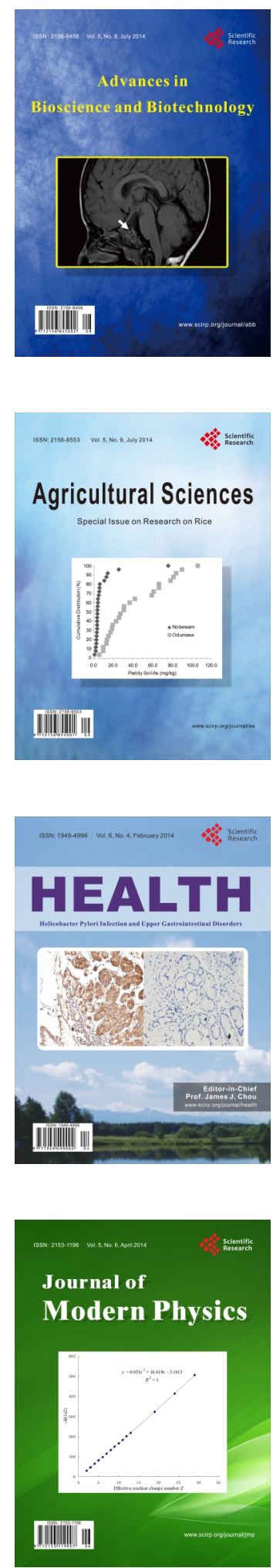
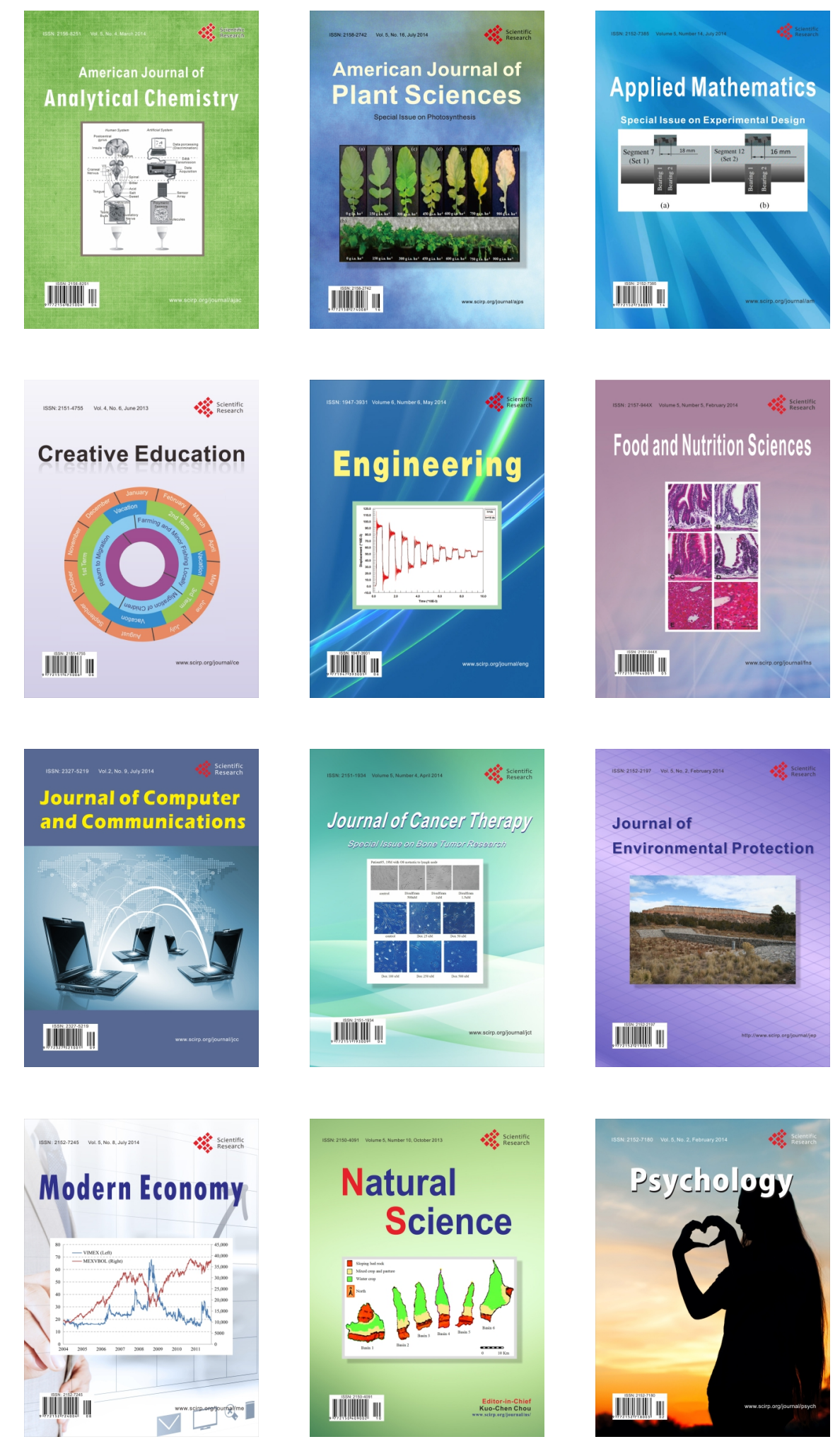